\title{
Two scenarios for avalanche dynamics in inclined granular layers
}

\author{
Tamás Börzsönyi, ${ }^{1,2, *}$ Thomas C. Halsey, ${ }^{3}$ and Robert E. Ecke ${ }^{1}$ \\ ${ }^{1}$ Condensed Matter and Thermal Physics \& Center for Nonlinear Studies, Los Alamos National Lab, NM, 87545, USA \\ ${ }^{2}$ Research Institute for Solid State Physics and Optics, POB 49, H-1525 Budapest, Hungary \\ ${ }^{3}$ ExxonMobil Research and Engineering, 1545 Route 22 East, Annandale, NJ 08801, USA
}

(Dated: November 7, 2018)

\begin{abstract}
We report experimental measurements of avalanche behavior of thin granular layers on an inclined plane for low volume flow rate. The dynamical properties of avalanches were quantitatively and qualitatively different for smooth glass beads compared to irregular granular materials such as sand. Two scenarios for granular avalanches on an incline are identified and a theoretical explanation for these different scenarios is developed based on a depth-averaged approach that takes into account the differing rheologies of the granular materials.
\end{abstract}

PACS numbers: $45.70 . \mathrm{Ht}, 45.70 .-\mathrm{n}, 45.70 . \mathrm{Mg}$

Avalanche behavior of granular material has many natural realizations from snow avalanches to massive rockslides. Physics-oriented avalanche investigations focusing on sand-pile kinematics [1] have given way to more recent work, which has probed granular interactions and the appropriate balance between continuum and discrete approaches [2]. Often avalanche dynamics have been studied with a bulk granular substrate, either in a rotating drum [1, 3, 4, 5, 6, 7] where the critical angle $\theta_{c}$, i.e., the angle where grains start to flow, is reached by the slow rotation of the drum, or on a pile 1,8$]$. Alternatively, one can investigate flow on an inclined plane where an underlying solid surface constrains the flow. In inclined layer granular flow at high volume flow rates a uniformly thick layer forms, whereas for lower flow rates waves in the form of thickness variations appear [9, 10, 11, 12, 13, 14]. For both the freely flowing and wave modulated cases, continuum descriptions of the flows based on flow rheology describe the experiments well [15, 16, 17.

In this Letter we focus not on steady or modulated flows but rather on flows exhibiting distinct, well-resolved avalanches. We have explored these flows on an inclined plane and have discovered two distinct scenarios for the dynamic avalanche behavior, depending on the character of the grains. For rough non-spherical grains (RNSG), avalanches are faster, bigger and overturning. Individual grains have down-slope speeds that exceed the front speed. By contrast, avalanches of spherical glass beads (SGB) are quantitatively slower and smaller, and the particles always travel slower than the front speed. We show that this difference in behavior arises from the differing rheologies of the different particle types in steady-state flow, and is linked to the stability of such flows. A theory based on the nonlinear properties of the depth-averaged equations makes quantitative predictions that agree with the experimental results and suggests that our different avalanche structures are related to the difference between roll waves and flood waves in hydrodynamic contexts [19]. In particular, the propagation of the RNSG avalanches involves a shock, while the SGB avalanches have a con- tinuous structure.

Two key lessons emerge from this study. The first is that our understanding of the rheology of dense granular flows is now sufficiently robust to allow successful modeling of quite detailed dynamical phenomena such as avalanche profiles. The second is that the quantitative differences between the rheology of the most commonly studied spherical particles and the more realistic rough particles result in qualitative, indeed, dramatic differences in their dynamical behavior.

A thin layer of granular material inclined at an angle $\theta$ is stable for a wide range of $\theta$ and thickness $h$. The onset of flow is expected only above a critical layer thickness 20, 21] $h_{c}$, and the flow subsides at $h_{s}<h_{c}$. The values of $h_{s}(\theta)$ and $h_{c}(\theta)$ decrease rapidly with increasing $\theta$. Thus, the layer can become unstable by increasing $\theta$ by a small amount $\delta \theta[9,18]$ or by increasing $h$ by adding new grains to the layer at a low flow rate, as we do here. In the former case, where the whole layer becomes metastable 9, 18, the shape and propagation of the avalanches depends critically on $\delta \theta$ such that either strictly downward or simultaneous downward and upward expanding avalanches can occur depending on the magnitude of $\delta \theta$. In our case a homogeneous static layer is prepared that is stable to small perturbations. New grains are only added at the top region $(5 \%)$ of the plane in the form of a very low incoming flux (shower) perpendicular to the plane. Due to the low incoming flux the height slowly increases locally, and when the little pile formed in this manner becomes unstable an avalanche is created, which travels down the rest of the plane on top of the stable static layer.

The experiments reported here used an inclined plane $2.2 \mathrm{~m}$ long and $0.4 \mathrm{~m}$ wide with a rough surface of particles glued to a glass substrate or sandpaper with different grit sizes. The grains were of two types: RNSG of sand or salt with several mean diameters $d$ (sand: $0.4 \pm 0.1$ $\mathrm{mm}$; sand: $0.2 \pm 0.1 \mathrm{~mm}$; salt: $0.4 \pm 0.1 \mathrm{~mm})$ and SGB with $d=0.5 \pm 0.1 \mathrm{~mm}$. The mass flow rate per unit width of the channel $Q$ was adjusted so that individual 
well-resolved avalanches formed, and was $0.17 \mathrm{~g} / \mathrm{s}-\mathrm{cm}$ for RNSG and $0.05 \mathrm{~g} / \mathrm{s}-\mathrm{cm}$ for SGB. Varying the incoming flux or the kinetic energy of the incoming particles gives rise to to a change in the size distribution and frequency of avalanches, which is not the subject of the present study. The angle $\theta$ was varied between $32^{\circ}$ and $41^{\circ}$ for RNSG and between $22^{\circ}$ and $26^{\circ}$ for SGB. The differences in $Q$ and $\theta$ for the different materials reflect the material variations in the critical angle $\theta_{c}$ and the angle of repose $\theta_{r}$ where flow stops. The experimental methods for obtaining the data presented below include: visualization of grain motion with high speed (1000fps) video imaging, determination of the lateral sizes of avalanches by image differencing, reconstruction of the $2 \mathrm{D}$ height profiles of avalanches using a laser sheet, and determination of particle and front velocities by the analysis of single particle trajectories on space-time plots.

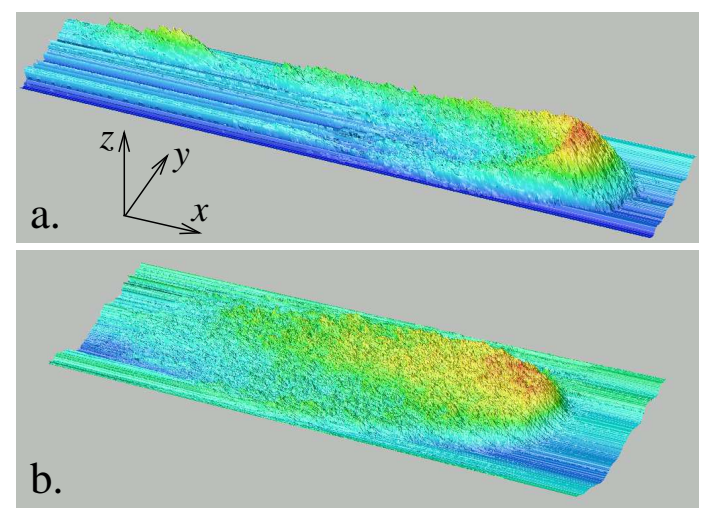

FIG. 1: Height profiles of a) RNSG avalanche for $\theta=36.8^{\circ}$. Image size $7.2 \mathrm{~cm} \times 56 \mathrm{~cm}$, (vertical size rescaled by $25 \mathrm{x}$ ) maximum height: $h_{m}=0.34 \mathrm{~cm}$, static layer thickness $h_{s}=$ $0.12 \mathrm{~cm}$; b) SGB avalanche for $\theta=24.3^{\circ}$. Image size $12.2 \mathrm{~cm}$ x $46.8 \mathrm{~cm}$, (vertical size rescaled by $25 \mathrm{x}$ ) maximum height: $h_{m}=0.29 \mathrm{~cm}$, static layer thickness $h_{s}=0.18 \mathrm{~cm}$.

Figure 1 shows the reconstructed 2D height profiles for sand and glass bead avalanches for operating conditions $\left(\theta=36.8^{\circ}, 24.3^{\circ}\right.$ and $Q=0.17 \mathrm{~g} / \mathrm{s}-\mathrm{cm}, 0.05 \mathrm{~g} / \mathrm{s}-\mathrm{cm}$ for RNSG and SGB, respectively) that produce roughly the same number of spatially-localized avalanches in an instantaneous image. For gravity-driven flows on an incline 22] the characteristic length scale is the height of the layer $h_{s}$ and the corresponding velocity scale is $\sqrt{g h_{s} \cos \theta}$. This normalization collapses data for different sized RNSG as shown in Fig. 2 where the spread is about $25 \%$ around the mean of the three data sets. For the SGB the velocities of avalanches with the same dimensionless areas are smaller by roughly a factor of 4. Further, the maximum dimensionless area of SGB avalanches is less than for RNSG avalanches, again by about a factor of 4 . The corresponding dimensionless avalanche velocity as a function of avalanche height, normalized by $h_{s}$, is shown in Fig. 2p. The data support the hypothesis that there are two classes of avalanches:
SGB avalanches have maximum height $h_{m}$ that is always less than $2 h_{s}$ whereas the height of RNSG avalanches is always greater than $2 h_{s}$. Our measurement of the height of avalanches relative to $h_{s}$ for SGB $h_{m} / h_{s} \approx 1.45 \pm 0.1$ is very similar to previous measurements for granular waves [9] for which $h_{m} / h_{s} \approx 1.55 \pm 0.1$, whereas the RNSG heights reported here are considerably higher: $h_{m} / h_{s} \approx 2.5 \pm 0.2$.
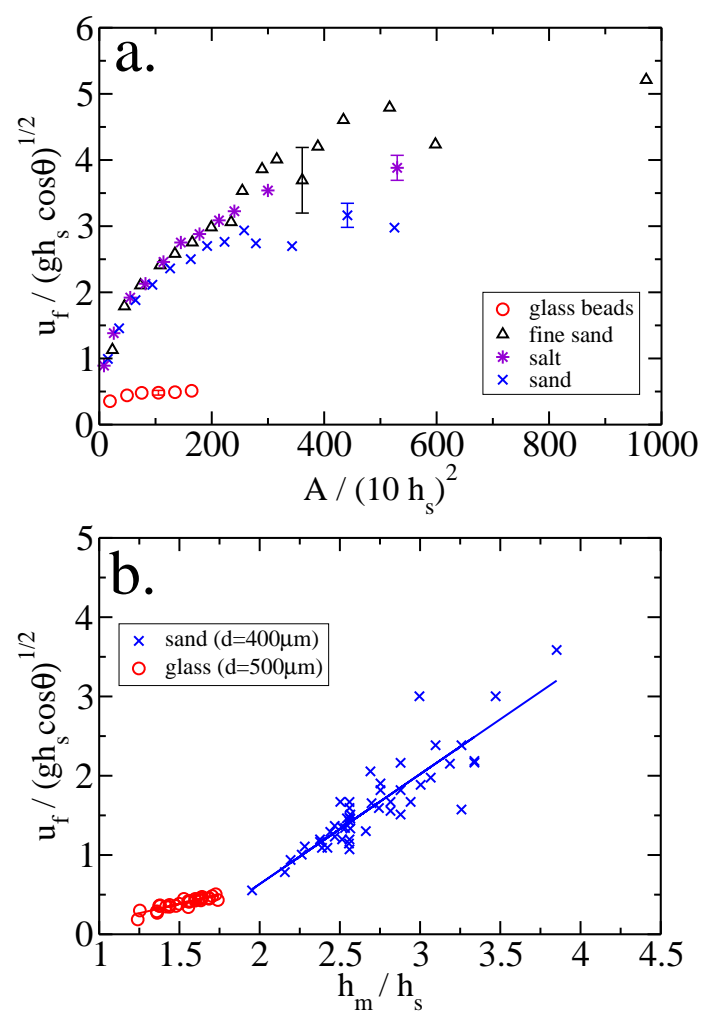

FIG. 2: Dimensionless avalanche front velocity vs a) dimensionless area A and b) dimensionless maximum height $h_{m} / h_{s}$ for sand $(\mathrm{x})$, glass beads $(\mathrm{o})$, salt $(*)$ and fine sand $(\triangle)$.

The origin of this dramatic difference is revealed in detailed measurements of the velocity distribution of surface particles for the two types of avalanches. The avalanche front speed $u_{f}$ and the surface mean particle speed $u_{p}$ right behind the front can be determined by measuring the image intensity along the centerline of an avalanche. Plotting the intensity in a space-time plot, see Fig. 3. reveals streaks associated with particles and a well-differentiated front for each avalanche type. For RNSG avalanches (Fig. 3a) the streaks within the avalanche are less steep than the front line, indicating $u_{p}>u_{f}$, whereas the opposite is true (Fig. 3 $\mathrm{b}$ ) for the SGB avalanches. In Fig. [4 the plot of $u_{p}$ versus $u_{f}$ shows that for SGB $u_{p}<u_{f}$, with a ratio $u_{p} / u_{f} \approx 0.7$, whereas for RNSG avalanches $u_{p}>u_{f}$ with $u_{p} / u_{f} \approx 1$.4. Another feature of the avalanches seen in Fig. 3 is the continuous form of SGB avalanches as evidenced by the curved paths just ahead of the front (representing the accelera- 
tion of particles in this region) as compared with particles being thrown out of the main body of the avalanche for the RNSG type. High speed imaging demonstrates 23] that an RNSG avalanche consists of a fast moving packet of grains that overtakes the front, like a breaking wave in a fluid, which entrains granular material from the underlying layer as it passes over. SGB avalanches, on the other hand, are continuous in that the static stresses that hold grains in place in front of the granular packet change and the previously stable packing collapses.

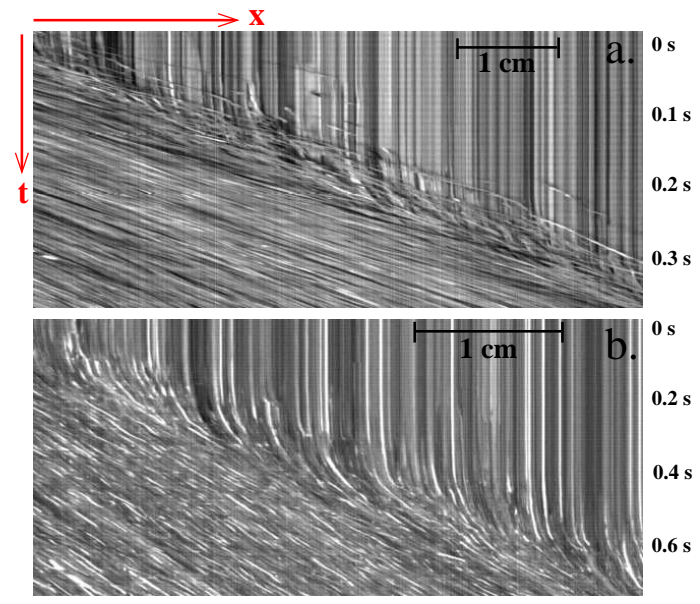

FIG. 3: Space-time plots taken along the symmetry axis of the avalanches a) sand for $\theta=36.8^{\circ}$ with scaled velocities (Froude numbers) $\operatorname{Fr}_{f}=2.12, \operatorname{Fr}_{p}=2.55$, and $\mathrm{b}$ ) glass beads $\theta=23.3^{\circ}$ with $\operatorname{Fr}_{f}=0.55, \operatorname{Fr}_{p}=0.33$.

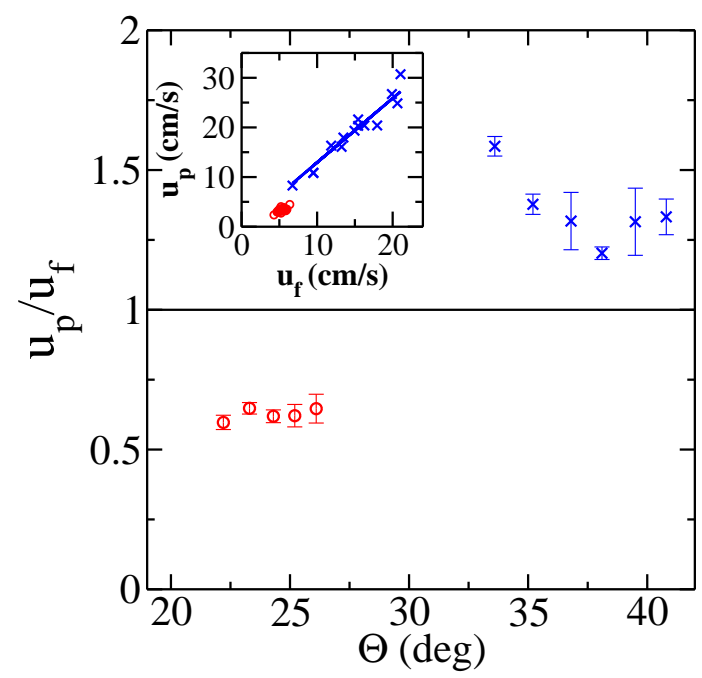

FIG. 4: Ratio of particle and front velocities $u_{p} / u_{f}$ for glass beads (o) and sand (x). The inset shows $u_{p}$ vs $u_{f}$ for $\theta=36.8^{\circ}$ (sand) and $\theta=25.2^{\circ}$ (glass beads).

These two distinct types of avalanches can be understood as consequences of the underlying rheology of the RNSG and SGB systems and of the structure of the dynamical equations for a propagating front. Let us first consider the rheology. For steady-state flows, both sand and glass beads have depth-averaged velocities $u$ given by the Pouliquen form 20, 22, 24]

$$
\frac{u}{\sqrt{g h}}=F\left(h / h_{s}(\theta)\right), \text { where } F(y)=\beta y-\gamma,
$$

where $h_{s}(\theta)$ is the minimum height of a flowing pile for an angle $\theta$. (Note that the depth-averaged quantities of the continuum model are different than the surface velocities measured in the experiments.) For sand [10], the coefficients in the function $F(y)$ are $\beta \approx \gamma \approx 0.7$, whereas for glass beads, $\beta \approx 0.14$ and $\gamma \approx 0$ [10, 20, 24]. We now apply these results to our RNSG and SGB systems, respectively.

The simplest dynamical picture of granular flows down an incline is achieved using the Saint-Venant shallow flow equations, adapted for granular media by Savage and Hutter 25]. For a flow of height $h$ and mean velocity $u$, these describe flow down a plane, with the plane parallel to the $x$-direction, by the averaged equations

$$
\frac{\partial h}{\partial t}+\frac{\partial(h u)}{\partial x}=0
$$

$$
\frac{\partial(h u)}{\partial t}+\alpha \frac{\partial\left(h u^{2}\right)}{\partial x}=\left(\tan \theta-\mu(u, h)-K \frac{\partial h}{\partial x}\right) g h \cos \theta
$$

Here $\alpha$ is determined by the profile of the flow, $\alpha=1$ for plug flow (as in Ref. [25]), $\alpha=4 / 3$ for a linear flow profile, or $5 / 4$ for a convex Bagnold profile 16 . The parameter $K$ is determined by the ratio of the normal stresses in the flow: the stress parallel to the bed, $\sigma_{x x}$, and that perpendicular to the bed, $\sigma_{z z}$. Numerical results show that $K \equiv \sigma_{x x} / \sigma_{z z} \approx 1$ for steady-state flows [24]. The friction coefficient $\mu(u, h)$ is determined by the requirement that the steady flow obey the rheology shown in Eq. (1), and will thus vary with the particle type.

Since the dimensionless velocity, typically given as the Froude number $\operatorname{Fr}=u / \sqrt{g h \cos \theta}$, is small near the critical angle or angle of repose for both the sand and the glass bead flows, it is natural to take the limit $\mathrm{Fr} \rightarrow 0$ in Eqs. (2] 3), which suppresses the LHS of Eq. (3). We thereby obtain with some work an approximate equation for $h$ :

$$
\frac{\partial h}{\partial t}+a(h) \frac{\partial h}{\partial x}=\nu \frac{\partial^{2} h}{\partial x^{2}} ; \quad a(h)=\sqrt{g h}\left(\frac{5}{2} \beta \frac{h}{h_{s}(\theta)}-\frac{3}{2} \gamma\right),
$$

where the "viscosity" $\nu$ can be computed as $\nu \sim d \sqrt{g h}$ [26]. This equation has solutions similar to those of Burger's equation, with a Burger's shock smoothed by the influence of the viscosity term. Thus, there is a solution consisting of a single hump propagating down the 
slope with velocity $a(h)$, with a smooth structure determined by the competition between this nonlinear velocity term on the LHS of Eq. (4) and the viscosity term.

Turning to the full system of Eqs. (2 3), however, we observe a potential flaw in this approach 19]. This full system is hyperbolic with characteristic velocities

$$
c_{ \pm}=u\left(\alpha \pm \sqrt{\alpha(\alpha-1)+\frac{K}{\mathrm{Fr}^{2}}}\right)
$$

If the velocity appearing in Eq. (4) does not obey $a<c_{+}$, then Eq. (4) predicts a structure that moves faster than the maximum rate at which information can be propagated in the full system of equations, which is clearly impossible. In these circumstances, the Burger's type solution transforms itself into a truly discontinuous solution traveling at velocity $c_{+}$[19], which is described by the full system Eqs. (2-3) rather than by Eq. (4).

Using the rheology determined by Eqs. (11), one can see that for SGB, taking a value of $\alpha=4 / 3$, this discontinuous solution will only develop for heights above $h / h_{s} \approx 6$, whereas for RNSG, it will develop as soon as the avalanche has a height $h / h_{s} \approx 1.3$ (the precise threshold depending on the value of $\theta$ ). This quantitative height condition is consistent with the data in Fig. 2] where $h_{m} / h_{s}<6$ for SGB and $h_{m} / h_{s}>1.3$ for RNSG. Further, calculation of the experimental ratio $a / c_{+}$using the rheology of Eqs. (1) yields $a / c_{+}<0.75$ for SGB and $a / c_{+}>1.1$ for RNSG, which is also consistent with this picture. Thus, we conclude that the glass bead avalanches reflect smooth solutions of Eq. (4), with $a<c_{+}$, whereas the sand avalanches represent discontinuous solutions of the full system, traveling at velocity $c_{+}$. The latter avalanches propagate into a quiescent bed because they are traveling at the characteristic velocity for the medium. The glass bead avalanches are analogous to "flood waves" in river flows, whereas the sand avalanches are analogous to "roll waves" in these flows 19, 27]. Note that ahead of the flowing avalanche, the moving material propagates into a material at rest, which is presumably in a state close to the critical Mohr-Coulomb state [28]. Unlike the flowing state, for which $\sigma_{x x} \approx \sigma_{z z}$, in this critical state $\sigma_{x x}>\sigma_{z z}$. Thus the transition region, in which the flow accelerates from rest into a pseudo-steady state described by the continuum theory, can be viewed as a region of passive Rankine failure, through which the compressive stress parallel to the bed, $\sigma_{x x}$, is decreasing with time. The mechanics of this region is complex, and cannot be described by the Saint-Venant equations alone.

Finally, we point out that in the linear theory of the instability of steady flows, developed by Forterre and Pouliquen, the criterion $a<c_{+}$corresponds to the stable regime of these flows with respect to wave disturbances [10]. Thus our observation of discontinuous avalanches for sand and smooth avalanches for glass beads dovetails nicely with their observation that steady flows of sand are far more unstable to such disturbances than are steady flows of glass beads.

We thank Brent Daniel, Michael Rivera, Ellák Somfai, and Ben White for helpful scientific conversations. Experimental work was funded by the US Department of Energy (W-7405-ENG-36).

* Electronic address: btamas@szfki.hu

[1] H.M. Jaeger, Chu-heng Liu and S.R. Nagel, Phys. Rev. Lett. 62, 40 (1989).

[2] J. Gollub, Physics Today 56, 10 (2003).

[3] J. Rajchenbach, Phys. Rev. Lett. 65, 2221 (1990).

[4] S. Courrech du Pont, P. Gondret, B. Perrin, and M. Rabaud, Phys. Rev. Lett. 90, 044301 (2003).

[5] P. Evesque, Phys. Rev. A 43, 2720 (1991).

[6] J. Rajchenbach, Phys. Rev. Lett. 88, 014301 (2002).

[7] P. Tegzes, T. Vicsek, and P. Schiffer, Phys. Rev. Lett. 89, 094301 (2002); Phys. Rev. E. 67, 051303 (2003).

[8] P.-A. Lemieux and D.J. Durian, Phys. Rev. Lett. 85, 4273 (2000).

[9] A. Daerr, Phys. of Fluids 13, 2115 (2001).

[10] Y. Forterre and O. Pouliquen, J. Fluid Mech. 486, 21 (2003).

[11] S.B. Savage, J. Fluid Mech. 92, 53. (1979).

[12] R.H. Davies, J. Hydrol. (NZ) 29, 18 (1990).

[13] S.N. Prasad, D. Pal and M.J.M. Römkens, J. Fluid Mech. 413, 89 (2000).

[14] M.Y. Louge and S.C. Keast, Phys. Fluids 13, 1213 (2001).

[15] J. Rajchenbach, Phys. Rev. Lett. 89, 074301 (2002).

[16] S. Douady, B. Andreotti and A Daerr, Eur. Phys. J. B. 11, 131 (1999).

[17] T. Emig, P. Claudin and J.P. Bouchaud, Europhys. Lett 50, 594 (2000).

[18] A. Daerr, and S. Douady, Nature 399, 241 (1999).

[19] G.B. Whitham, Linear and Nonlinear Waves (Wiley, New York, 1999).

[20] O. Pouliquen, Phys. of Fluids 11, No.3, 542 (1999).

[21] O. Pouliquen and N. Reanut, J. Phys. II. France 6, 923 (1996).

[22] D. Ertas, and T.C. Halsey, Europhys. Letts. 60, 931 (2002).

[23] Movies of avalanches taken with a high speed camera can be downloaded from:

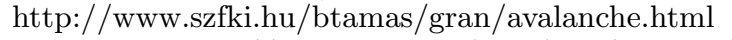
or http://www.lanl.gov/orgs/mst/MST10/fluid dynamics/granular.shtml

[24] L.E. Silbert, D. Ertas, G.S. Grest, T.C. Halsey, D. Levine and S.J. Plimpton, Phys. Rev. E 64, 051302-1, (2001).

[25] S.B. Savage and K. Hutter, J. Fluid Mech. 199, 177 (1989).

[26] Physical arguments based on the theory of ref. 22] suggest that in a more complete description, $\nu \approx h_{s} \sqrt{g h}$; O. Pouliquen, private communication.

[27] R.F. Dressler, Comm. Pure Appl. Math.2, 149 (1949).

[28] R.M. Nedderman, Statics and Kinematics of Granular Materials (Cambridge Univ. Press, 1992, New York) Chapter 2. 